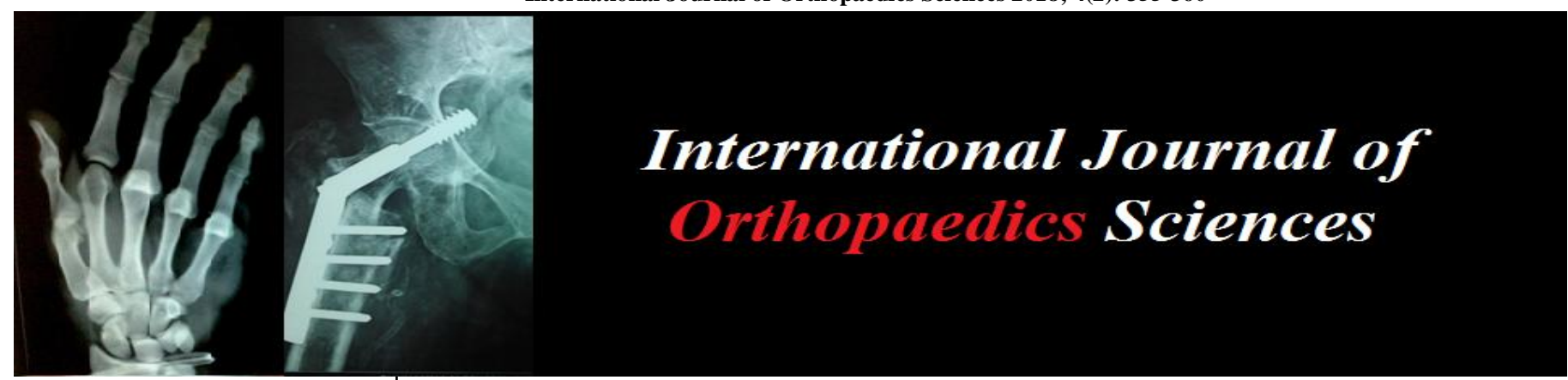

ISSN: $2395-1958$

IJOS 2018; 4(2): 355-360

(C) 2018 IJOS

www.orthopaper.com

Received: 15-02-2018

Accepted: 17-03-2018

Naser M Selim

Assistant Prof. of Orthopedic Surgery Knee Surgery -

Arthroscopy and Sports Injuries Unit Orthopedic Department

Mansoura University
Correspondence

Naser M Selim

Assistant Prof. of Orthopedic

Surgery Knee Surgery -

Arthroscopy and Sports Injuries

Unit Orthopedic Department

Mansoura University

\section{Conjoint tendon transfer: Tenodesis for coracoclavicular ligaments reconstruction in acute high grade acromioclavicular dislocation}

\section{Naser M Selim}

DOI: https://doi.org/10.22271/ortho.2018.v4.i2f.55

\begin{abstract}
Poor long-term outcomes after Dewar and Barington technique (1965) in the treatment of acromioclavicular joint (ACJ) dislocation were reported. Its modification by conjoint tendon transfer tenodesis may yield stable and anatomic reduction. The purpose of this study is to evaluate the clinical and radiological results of patients with acute high-grade acromioclavicular joint (ACJ) dislocation treated with conjoint tendon transfer - tenodesis. Thirty-two patients were operated in the period between March 2012 and June 2015 and could be recruited and evaluated at the final follow-up. Preoperative documentation of coracoclavicular distance, VAS (visual analogue scale) for pain, ASES (American Shoulder and Elbow Surgeons) score and Constant score was done. Reconstruction was performed using conjoint tendon transfer - tenodesis. Statistical analysis was done using the statistical package for social science (SPSS) program version 20.0. At a median follow-up of 34.4 (range 24.3-60.2) months after the procedure, the coracoclavicular distance decreased significantly from a mean of $21.5 \mathrm{~mm}$ preoperatively to a mean of $11.5 \mathrm{~mm}$ postoperatively $(\mathrm{P}<0.001)$. The VAS for pain improved significantly from an average of 8.5 points preoperatively to 1.2 at the last follow-up $(\mathrm{P}<0.001)$. Constant score and ASES score improved significantly from 70.69 and 65 preoperatively to 95.25 and 96.4 respectively at the last follow-up ( $\mathrm{P}<0.001$ ). A deep infection affected one patient. The study concluded that, coracoclavicular ligaments reconstruction by conjoint tendon transfer - tenodesis technique achieves excellent results and could be considered a valid surgical option of management in acute high grade acromioclavicular dislocations.
\end{abstract}

Keywords: conjoint tendon - coracoclavicular ligaments reconstruction - acromioclavicular dislocation

\section{Introduction}

There was no ideal surgical technique for treatment of acromioclavicular joint (ACJ) dislocations ${ }^{[1-3]}$. The surgical techniques varied between fixation ${ }^{[4-9]}$ and coracoclavicular ligaments (CCL) repair, reconstruction, substitution ${ }^{[10-12]}$ or ligament / tendon graft transfer ${ }^{[13-}$ 15-19, 20-22-27]

Surgical techniques for fixation of ACJ dislocation were associated with a high incidence of early and late postoperative complications $[28,29]$. While CCL repair, reconstruction or substitution showed relatively better results and had evolved over the last several decades ${ }^{[10-12 \text {, }}$ 30- 32].

It was found that the conjoint tendon has better properties and greater consistency of quality compared with those of coracoacromial ligament ${ }^{[14,21,25]}$. The conjoint tendon is not as strong as the native CCL, but it is stronger than the commonly used coracoacromial ligament ${ }^{[25]}$.

Dewar and Barington procedure (1965) had transferred the conjoint tendon to the lateral clavicle creating a dynamic stabilization of the lateral clavicle ${ }^{[18]}$. But the long-term outcomes were poor ${ }^{[19]}$.

This prospective study describes a modification of Dewar and Barington technique by conjoint tendon transfer-tenodesis for the CCL reconstruction and assesses its results in the treatment of an acute high grade ACJ dislocation. 


\section{Patients and Methods}

The study is a case series of level IV evidence. It is accepted by Institutional Review Board (IRB). The study was carried on thirty-six patients in the period between March 2012 and June 2015. It included acute (up to 3 weeks of trauma), Rockwood's type IV: VI ACJ dislocation and excluded chronic or open or lower grades dislocations.

Thirty -two out of thirty-six patients were collected at the final follow up and were available for clinical and radiological evaluation. Four patients were not available for follow-up evaluation. Of the 32 patients; 24 were males and 8 females (3:1), with average age 32 years, 12 were exposed to road traffic accidents and 20 were fall from a height on the affected shoulder. ACJ dislocation affected the dominant side in 20 patients and the non-dominant side in 12 patients. ACJ dislocations were grade IV in 18 patients and grade $\mathrm{V}$ in 14 patients (Table 1).

All patients were operated after one week of trauma. The preoperative VAS for pain, ASES, Constant score and assessment of biceps power and examination for musculocutanous nerve and measurement of the coracoclavicular distance on plain X-ray were recorded.

All patients were treated by CCL reconstruction using conjoint tendon transfer- tenodesis. Postoperative shoulder immobilization for six weeks was done. Then active elevation was started gradually by $45^{\circ}$ increase every week till full elevation. Then muscle strengthening of the shoulder was done. The clinical and radiological results were recorded at the final follow up.

Table 1: Patient demography

\begin{tabular}{|c|c|c|c|}
\hline \multicolumn{2}{|c|}{ Age } & 32.00 & \pm 6.86 \\
\hline \multirow{2}{*}{ Gender } & female & 8 & $25.0 \%$ \\
\hline & male & 24 & $75.0 \%$ \\
\hline \multirow{2}{*}{ Affected side } & left & 12 & $37.5 \%$ \\
\hline & right & 20 & $62.5 \%$ \\
\hline Dominant side & right & 32 & $100 \%$ \\
\hline \multirow{2}{*}{ Mechanism of injury } & fall down & 20 & $62.5 \%$ \\
\hline & traffic accident & 12 & $37.5 \%$ \\
\hline \multirow{2}{*}{ Grade of dislocation } & IV & 18 & $56.3 \%$ \\
\hline & $\mathrm{V}$ & 14 & $43.7 \%$ \\
\hline
\end{tabular}

\subsection{Surgical technique}

Under general anesthesia and beach chair position, a skin incision is made from just posterior to the ACJ to about $2 \mathrm{~cm}$ distal to the tip of coracoid process. The anterior aspect of the deltoid is split for up to $4 \mathrm{~cm}$ distally in the direction of its fibers with detachment of a part of deltoid origin from the clavicle. The conjoint tendon is exposed at its attachment to the tip of the coracoid process. The torn CCL are identified (Fig. 1).
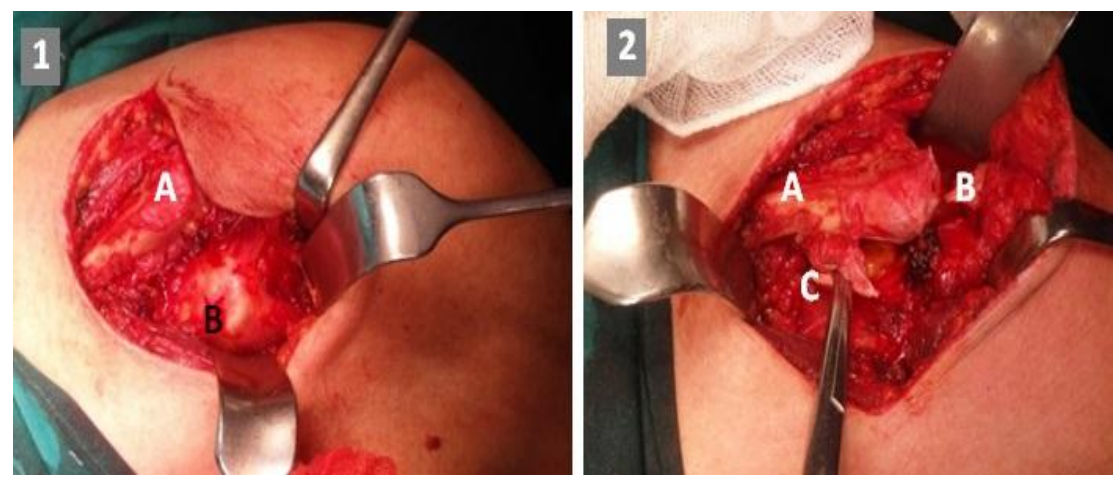

Fig 1: surgical exposure of ACJ of the left shoulder: fig1-1 shows the lateral part of the clavicle (A) and the coracoid process with its attachments (B). Fig1-2 shows the lateral part of the clavicle (A), the ACJ before reduction (B) and the torn CCL (C).

2.1.1 Preparation of the clavicle: debridement of the under surface of the clavicle is done and a row bed at attachment of CCL is created with a burr. Two clavicular holes are drilled by $1.7 \mathrm{~mm}$ drill bit. The first hole is drilled at mid clavicle or slightly anterior and $3 \mathrm{~cm}$ from the lateral end of the clavicle (i.e. trapezoid hole). The second hole is drilled posterior to the first one, $7 \mathrm{~mm}$ anterior to conoid tubercle and $4.5 \mathrm{~cm}$ from the lateral end of the clavicle (i.e. conoid hole).

2.1.2 Coracoid osteotomy: before the osteotomy, the conjoint tendon is tagged by Ethibond suture no 2 (Johnson \& Johnson) and a transverse hole is drilled in the coracoid process from lateral to medial by $1.7 \mathrm{~mm}$ drill bit just posterior to coracoid tip. Then by electocautery pencil, a line for planned osteotomy site is determined extending from anterolateral to posteromedial part of coracoid process.

Osteotomy is done by sharp osteotome (Fig. 2-1). The coracoacromial ligament is preserved laterally and the neurovascular structures are protected medially. After osteotomy, and by a small bone nibbling forceps, a part of the center of coracoid bed is removed forming a socket to embrace the transferred conjoint tendon. A coracoid hole is drilled from anteromedial to posterolateral direction in the coracoid socket using $2.5 \mathrm{~mm}$ drill bit.
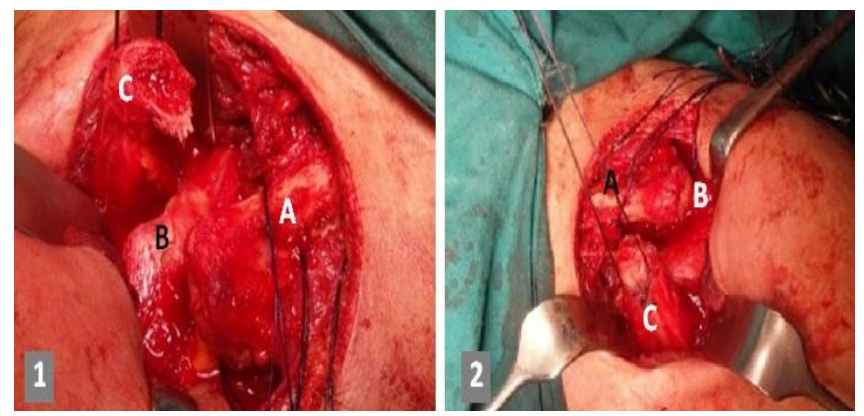

Fig 2: coracoid osteotomy and conjoint tendon mobilization: fig2-1: top view of left shoulder shows the lateral part of the clavicle (A) and the coracoid bed after osteotomy (B) and conjoint tendon with the tip of coracoid (C). Fig2-2: frontal view of left shoulder shows the lateral part of the clavicle (A), the ACJ before reduction (B) and conjoint tendon mobilization (C).

2.1.3 Mobilization: with the Ethibond suture tagged in the conjoint tendon, gentle mobilization is done while the elbow and shoulder flexed till the tendon can reach the under surface of the clavicle (Fig. 2-2). Debridement of the conjoint tendon 
and removal of muscle tissues are done. The musculocutaneous nerve enters the coracobrachialis muscle $5-8 \mathrm{~cm}$ below the coracoid process but there are many variations.

The Ethibond sutures in conjoint tendon are passed through clavicular holes. $1.5 \mathrm{~mm}$ cerclage wire is passed through conoid hole of clavicle then through the transverse hole of coracoid fragment from medial to lateral and finally through the trapezoid hole of the clavicle. This is followed by approximation of the coracoid tip and the conjoint tendon to the under surface of the clavicle with proximal traction on the sutures, while part of the tendon is embraced in the coracoid socket between its medial and lateral borders distally (Fig. 3$1)$.
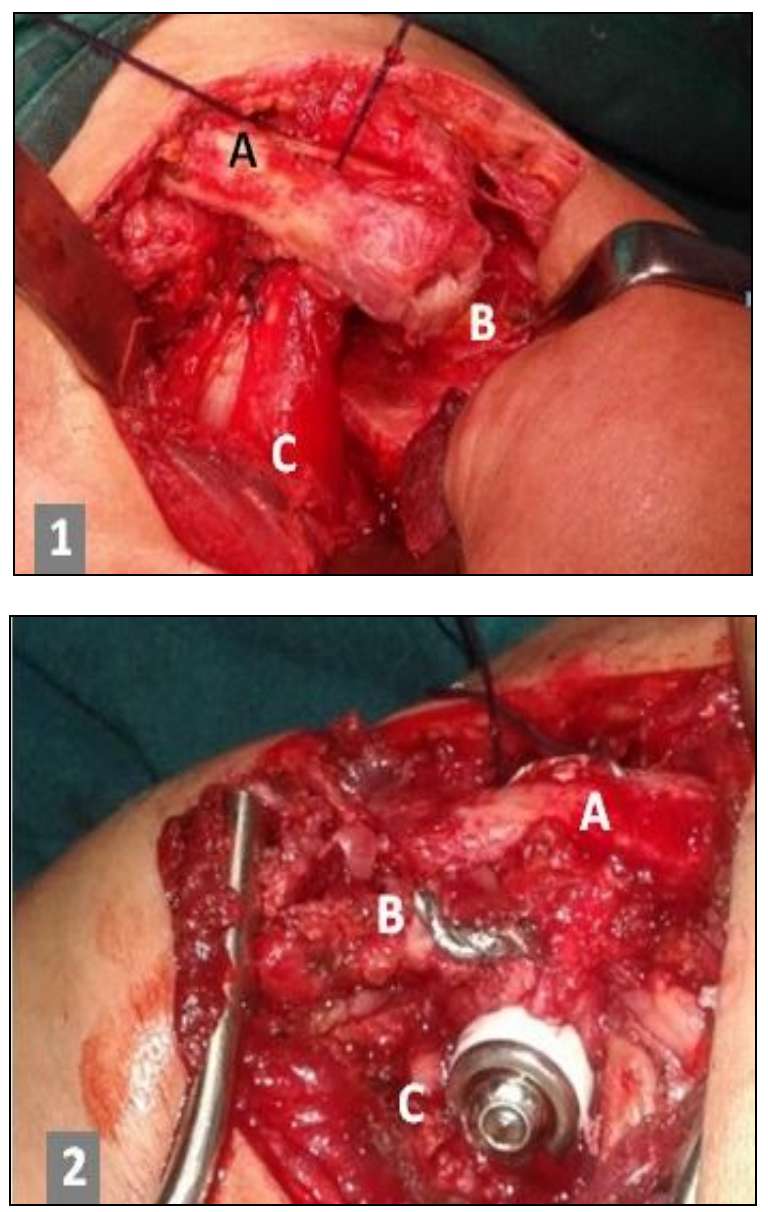

Fig 3: conjoint tendon transfer and fixation: fig3-1: frontal view of left shoulder shows the lateral part of the clavicle (A) and the ACJ before reduction (B) and transferred conjoint tendon to under surface of the clavicle (C). Fig3-2: frontal view of left shoulder shows the lateral part of the clavicle (A) after reduction and conjoint tendon fixation by cerclage wire (B) and tenodesis to coracoid bed by screw and washer.

2.1.4 Reduction and fixation: ACJ is reduced. The coracoid tip and conjoint tendon are pulled upwards, rotated internally and fixed to under surface of the clavicle by the cerclage wire (Fig. 3-2). The fixation is done while the elbow and the shoulder flexed to relax the tendon. The position of clavicular holes allows anatomic proximal attachment of transferred tendon. Then the debrided conjoint tendon is tenodesed to the coracoid socket by $4 \mathrm{~mm}$ screw and plastic washer (Fig. 3-2) while the elbow and shoulder extended to tension the tendon. The deeper the coracoid socket, the nearer to coracoid base, the more anatomic is the distal attachment of transferred tendon. At the end the detached deltoid origin and the deltotrapezial fascia are repaired. Postoperative x-ray is done.

\subsection{Clinical Evaluation}

The clinical evaluation consisted of a complete physical examination of both shoulders and assessment of VAS for pain, ASES, Constant score and assessment of biceps power and examination for musculocutanous nerve.

\subsection{Radiological Evaluation}

For radiological evaluation of vertical translation, stress plain $\mathrm{X}$-ray views were obtained (Fig. 4). The CC distance on both sides was measured.
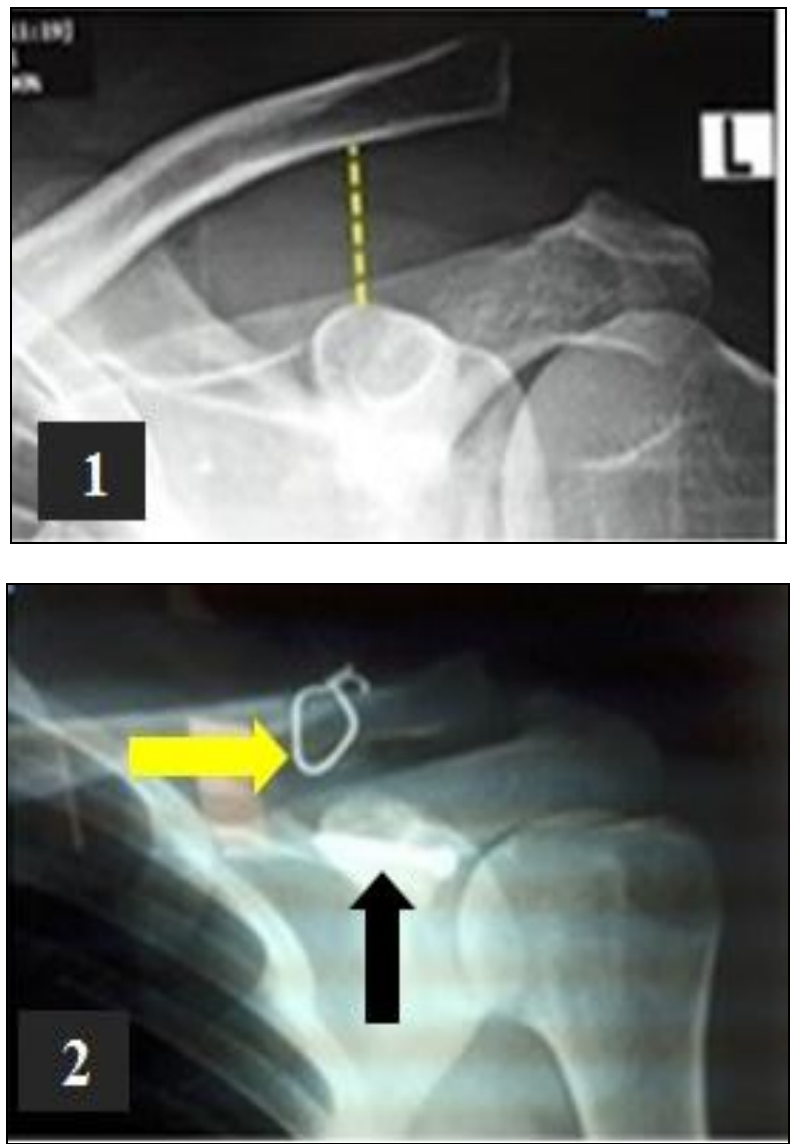

Fig 4: $x$-ray of left shoulder after conjoint tendon: fig.4-1 shows preoperative $\mathrm{x}$-ray with coracoclavicular distance (dotted yellow line $)=82.09$ pixel $=3.6 \mathrm{~cm}$. and fig. $4-2$ shows postoperative $\mathrm{x}$-ray with the coracoclavicular distance $=27$ pixel $=1.2 \mathrm{~cm}$. (calibrated normal distance: 27 pixel $=1.2 \mathrm{~cm}$ ) and also shows cerclage wire (yellow arrow) and metal screw (black arrow) fixation.

\subsection{Statistical Analysis}

Data were tabulated, coded then analyzed using the SPSS version 20. Descriptive statistics were calculated in the form of a mean \pm standard deviation (SD) for quantitative parametric data, median and range (minimum and maximum) for quantitative non-parametric data and frequency for qualitative data.

Analytical statistics were used in comparison between the different groups, the significance of difference was tested using one of the following tests: Student's t-test (Paired): used to compare between the mean of two related groups of numerical (parametric) data and Wilcoxon signed rank test: used to compare between two related groups of numerical (non-parametric) data. $\mathrm{P}$ value: $<0.05$ was considered statistically significant.

Digital radiographs were analyzed on Intel ${ }^{\circledR}$ Core $I 3 \AA$ based computer using Video Test Morphology® software (Russia) with a specific built-in routine for calibrated distance measurement. Free hand line tool used to measure the 
coracoclavicular distance. Results obtained in pixels were converted to centimeter $(\mathrm{cm})$ after being calibrated against normal radiograph (Fig. 4).

\section{Results}

At a minimum of two years follow up after the procedure. The postoperative VAS for pain, ASES, Constant score and assessment of biceps power and examination for musculocutanous nerve and measurement of the coracoclavicular distance on stress plain x-ray were recorded and statistically analyzed.

3.1 Clinical results: The VAS for pain improved significantly from an average of 8.5 points preoperatively to 1.5 at the last follow up $(\mathrm{P}<0.001)$. Constant and ASES scores improved significantly from a mean of 70.69 and 65 preoperatively to a mean of 95.25 and 96.4 respectively at the last follow up $(\mathrm{P}<0.001)$ (Table 2, 3). All patients were satisfied with the procedure to different degrees.

3.2 Radiological results: The preoperative coracoclavicular distance ranged from $18 \mathrm{~mm}$ to $36 \mathrm{~mm}$ with the mean of 21.5 $\mathrm{mm}$. Immediate postoperative radiographs confirmed adequate reduction of the ACJ in all 32 patients. The coracoclavicular distance ranged from $10 \mathrm{~mm}$ to $14 \mathrm{~mm}$ with the mean of 11.5 $\mathrm{mm}$ at the final follow up. There was a significant reduction of the coracoclavicular distance postoperatively $(\mathrm{P}<0.001)$ (Table 2, 3). There was no significant difference in the coracoclavicular distance between the operated and the nonoperated shoulder (mean $10.9 \mathrm{~mm}$ ).

3.3 Complications: a deep infection affected one patient. An open debridement and early removal of implants after 4 months of surgery were done to him. The patient still has stable ACJ and coracoclavicular distance remained unchanged at the last follow up. The CC difference of patients with implant removal did not differ significantly from those of patients without. The overall revision rate was $0 \%$.

The VAS score in the present study indicated high level of patient satisfaction. The functional and subjective results were excellent in all cases at the last follow up. There were no cases of nonunion between coracoid tip and undersurface of the clavicle, no cases had AC redislocation. There was no musculocutanous nerve injury and no effect on conjoint tendon function.

Table 2: Final postoperative findings

\begin{tabular}{|c|c|c|c|}
\hline \multicolumn{2}{|c|}{ Final Postoperative results } & Mean & SD \\
\hline \multicolumn{2}{|c|}{ Coracoclavicular distance $\mathrm{cm}($ mean $\pm \mathrm{SD})$} & 1.15 & .15 \\
\hline \multicolumn{2}{|c|}{ Constant sore $($ mean \pm SD) } & 95.25 & 1.91 \\
\hline \multicolumn{2}{|c|}{ ASES (mean \pm SD) } & 96.4 & 1.2 \\
\hline \multicolumn{2}{|c|}{ Pain VAS (median-range) } & 1.5 & $.00-2.00$ \\
\hline Biceps power & $\mathrm{V}$ & 32 & $100.0 \%$ \\
\hline Musculocutanous nerve & free & 32 & $100.0 \%$ \\
\hline \multirow{2}{*}{ Infection } & deep & 1 & $3.1 \%$ \\
\hline & no & 31 & $96.9 \%$ \\
\hline
\end{tabular}

Table 3: Comparison between preoperative and post-operative findings

\begin{tabular}{|c|c|c|c|c|}
\hline \multicolumn{2}{|c|}{ Pre and post-operative results } & Mean & \pm SD & $\mathbf{P}$ \\
\hline \multirow{2}{*}{$\begin{array}{c}\text { Coracoclavicular distance } \\
\mathrm{cm}\end{array}$} & Pre. & 2.15 & .23 & \multirow{2}{*}{$\begin{array}{c}< \\
0.001\end{array}$} \\
\hline & Post. & 1.15 & .15 & \\
\hline \multirow{2}{*}{ Constant score } & Pre. & 70.69 & 2.6 & \multirow{2}{*}{$\begin{array}{c}< \\
0.001\end{array}$} \\
\hline & Post. & 95.25 & 1.91 & \\
\hline \multirow{2}{*}{ ASES } & Pre. & 65 & 2.3 & \multirow{2}{*}{$\begin{array}{c}< \\
0.001 \\
\end{array}$} \\
\hline & Post. & 96.4 & 1.2 & \\
\hline & & Median & Range & $\mathrm{P}$ \\
\hline \multirow[t]{2}{*}{ Pain VAS } & Pre. & 8.5 & $\begin{array}{l}8.00- \\
10.00\end{array}$ & \multirow[t]{2}{*}{$<0.001$} \\
\hline & Post. & 1.5 & $.00-2.00$ & \\
\hline
\end{tabular}

$($ Pre $=$ preoperative. Post $=$ postoperative. SD: standard deviation P: Probability)

\section{Discussion}

Surgical techniques for fixation of ACJ dislocation are associated with high incidence of early and late postoperative complications. $32 \%$ technical failure rate after Bosworth screw fixation and $43 \%$ early postoperative complications and $32 \%$ late instability after tension band fixation were reported $[28,29]$.

$58 \%$ early postoperative complications and $50 \%$ late instability after kook plate fixation and $17 \%$ early postoperative complications and 34\% late instability after polydiaxone suture suspension were also reported [28, 29]. While CCL repair, reconstruction or substitution showed relatively better results and had evolved over the last several decades ${ }^{[10-12,30-32]}$.

Many surgeons use tendon grafts as semitendinosus or gracilis passed through coracoid and clavicular bone tunnels for CCL reconstructions with fixation by different methods of fixation [10-12, 30-32]. But tendon graft reconstructions are costly ${ }^{[10]}$, technically demanding may precipitate coracoid or clavicular fracture $[10,32]$ and may be associated with distant site morbidity, graft stretch, tunnel widening ${ }^{[31]}$ and infection.

Weaver -Dunn procedure (1972) comprises excision of the lateral end of clavicle and transfer of the coracoacromial ligament ${ }^{[27]}$. The procedure carries a risk of ongoing pain, recurrent instability. It has only $30 \%$ strength \& $10 \%$ stiffness of intact ligaments ${ }^{[20]}$. The mean laxity after the procedure is $42 \mathrm{~mm}$ in horizontal plane and $14 \mathrm{~mm}$ in vertical plane while it is $8 \mathrm{~mm}$ and $3 \mathrm{~mm}$, respectively in intact ligaments ${ }^{[17]}$. It is biomechanically inferior to tendon graft reconstructions [33] and could be augmented by either auto-graft or synthetic suture material ${ }^{[17,3]}$.

Sloan SM et al. (2004) found that the ultimate tensile strength of the intact CCL is $621+/-209 \mathrm{~N}$ and that of the lateral 12 $\mathrm{mm}$ of the conjoint tendon is $265+/-79 \mathrm{~N}$ and that of coracoacromial ligament is $246+/-69 \mathrm{~N}$. And they believe that the lateral half of the conjoint tendon is a viable alternative auto-graft source for CCL reconstruction ${ }^{[25]}$.

The conjoint tendon has better properties and greater consistency of quality of the graft compared with those of coracoacromial ligament ${ }^{[14,21,25]}$ though not as strong as the native CCL; the conjoint tendon is stronger than the commonly used coracoacromial ligament ${ }^{[25]}$.

Jiang et al. (2007) performed proximally based conjoint tendon transfer for CCL reconstruction that retains the origin of the tendon and divides its lateral half distally, then reverses 
the tendon and implants it into the lateral end of the clavicle and fix it using 2 suture anchors loaded with Ethibond sutures for augmentation. Jiang technique is costly, non-anatomic, changes the conjoint tendon into single tendon for coracobrachialis attachment and in addition, the anterior attachment of the transferred part to coracoid leads to anterior displacement of the clavicle and mal reduction ${ }^{[21,25]}$.

Dewar and Barington (1965) transferred the conjoint tendon to the lateral clavicle for CCL reconstruction. The transfer is either direct tendon transfer ${ }^{[26]}$ or together with tip of coracoid $[14,15]$. This creates a dynamic stabilization of the lateral clavicle. It has been used for the treatment of acute and chronic injuries, with better results in younger patients ${ }^{[18]}$.

But the procedure carries the risk of over-tightening of the coracoclavicular space, subsequent fracture of coracoid [13], nonunion of transferred coracoid, loss of fixation or screw breakage and injury of musculocutanous nerve ${ }^{[16,23]}$. Poor long-term outcomes were reported ${ }^{[19]}$.

In this study conjoint tendon transfer - tenodesis creates a static stabilization of the lateral clavicle. The technique consists of 4 steps: osteotomy of the coracoid then mobilization of conjoint tendon then transfer to the under surface of the clavicle and fixation by cerclage wire and tenodesis to bed of retained coracoid process and fixation by screw and plastic washer.

The technique creates anatomic clavicular CCL foot prints, depends on bone to bone healing at the clavicle and tendon to bone healing at the coracoid bed. It also creates nearly anatomic CCL coracoid foot prints. It is double bundle reconstruction where the short head of biceps represents the anterolateral bundle (trapezoid ligament) and the coracobrachialis represents the posteromedial bundle (conoid ligament) of CCL.

It gives secure stable fixation on the cases studied and it is done by simple and cheap method of fixation. It is done using small clavicular and coracoid holes and so no fractures or tunnel widening was recorded. Good tension of this local graft is obtained as the distal fixation is done with the elbow and shoulder extended.

The procedure has no effect on function of conjoint tendon as all studied patients have full biceps power. It preserve coracoacromial ligament and not associated with distant site morbidity. The early clinical and radiological results are promising and show significant improvement of radiological measurements used and of all clinical scores applied and all patients were satisfied with the procedure to different degrees.

\subsection{The points of weakness}

The number of patients is relatively small.

\section{Conclusions}

Although the study includes a relatively small number of cases of the CCL reconstruction by conjoint tendon transfer tenodesis technique, it gives excellent results that are promising to consider the technique as a valid option of management in acute high grades acromioclavicular dislocations.

\section{References}

1. Fukuda K, Craig EV, An KN, Cofield RH, Chao EY. Biomechanical study of the ligamentous system of the acromioclavicular joint. JBJS (Am). 1986; 68-A:434-40.

2. LaPrade RF, Hilger B. Coracoclavicular ligament reconstruction using a semitendinosus graft for failed acromioclavicular separation surgery. Arthroscopy. 2005; 21:1277e1-e5.

3. Mazzocca AD, Santangelo SA, Johnson ST, Rios CG, Dumonski ML, Arciero RA. A biomechanical evaluation of an anatomical coracoclavicular ligament reconstruction. Am J Sports Med. 2006; 34(2):236-46.

4. Bosworth BM. Acromioclavicular dislocation: endresults of screw suspension treatment. Ann Surg. 1948; 127(1):98-111.

5. Lancaster S, Horowitz M, Alonso J. Complete acromioclavicular separations: a comparison of operative methods. Clin Orthop Relat Res. 1987; 216:80-8.

6. Lizaur A, Marco L, Cebrian R. Acute dislocation of the acromioclavicular joint: Traumatic anatomy and the importance of deltoid and trapezius. JBJS (Br). 1994; 76(4):602-6.

7. McConnell AJ, Yoo DJ, Zdero R, Schemitsch EH, McKee MD. Methods of operative fixation of the acromioclavicular joint: a biomechanical comparison. J Orthop Trauma. 2007; 21:248-53.

8. Nadarajah R, Mahaluxmivala J, Amin A, Goodier DW. Clavicular hook-plate: complications of retaining the implant. Injury. 2005; 36(5):681-3.

9. Sim E, Schwarz N, Hocker K, Berzlanovich A. Repair of complete acromioclavicular separations using the acromioclavicular-hook plate. Clin Orthop Relat Res. 1995; 314:134-42.

10. Natera L, Reiriz JS, Abat F. Anatomic reconstruction of chronic coracoclavicular ligament tears: arthroscopicassisted approach with non-rigid mechanical fixation and graft augmentation. Arthrosc Tech. 2014; 3(5):e583-8.

11. Shetty NS, Yoo YS, Kim DY, Lee SS, Jeong US. Open anatomical coracoclavicular ligament reconstruction using a tendon graft with an endobutton loop. Acta Orthop Belg. 2009; 75(6):828-31.

12. Yoo YS, Seo YJ, Noh KC, Patro BP, Kim DY. Arthroscopically assisted anatomical coracoclavicular ligament reconstruction using tendon graft. Int Orthop (SICOT). 2011; 35(7):1025-30.

13. Baumgarten KM, Altchek DW, Cordasco FA. Arthroscopically assisted acromioclavicular joint reconstruction. Arthroscopy. 2006; 22(2):228.e1-e6.

14. Berson BL, Gilbert MS, Green S. Acromioclavicular dislocations: treatment by transfer of the conjoined tendon and distal end of the coracoid process to the clavicle. Clin Orthop Relat Res. 1978; 135:157-64.

15. Brunelli G, Brunelli F. The treatment of acromioclavicular dislocation by transfer of the short head of biceps. Int Orthop. 1988; 12(2):105-8.

16. Caspi I, Ezra E, Nerubay J, Horoszovski H. Musculocutanous nerve injury after coracoid process transfer for clavicle instability: report of three cases. Acta Orthop Scand. 1987; 58:294-5.

17. Deshmukh AV, Wilson DR, Zilberfarb JL, Perlmutter GS. Stability of acromioclavicular joint reconstruction: biomechanical testing of various surgical techniques in a cadaveric model. Am J Sports Med. 2004; 32(6):1492-8.

18. Dewar IT, Barrington TW. The treatment of chronic acromioclavicular dislocation. JBJS (Br). 1965; 47:32-5.

19. Ferris BD, Bhamra M, Paton DF. Coracoid process transfer for acromioclavicular dislocations: A report of 20 cases. Clin Orthop Relat Res. 1989; 242:I84-94.

20. Harris RI, Wallace AL, Harper GD, Goldberg JA, Sonnabend DH, Walsh WR. Structural properties of the intact and the reconstructed coracoclavicular ligament complex. Am J Sports Med. 2000; 28(1):103-8. 
21. Jiang C, Wang M, Rong G. Proximally based conjoined tendon transfer for coracoclavicular reconstruction in the treatment of acromioclavicular dislocation. JBJS (Am). 2007; 89(11):2408-12.

22. Lafosse L, Baier GP, Leuzinger J. Arthroscopic treatment of acute and chronic acromioclavicular joint dislocation. Arthroscopy. 2005; 21(8):1017.

23. Lemos MJ, Tolo ET. Complications of the treatment of the acromioclavicular and sternoclavicular joint injuries, including instability. Clin Sports Med. 2003; 22(2):37185.

24. Skjeldal S, Lundblad R, Dullerud R. Coracoid process transfer for acromioclavicular dislocation. Acta Orthop Scand. 1988; 59:180-2.

25. Sloan SM, Budoff JE, Hipp JA, Nguyen L. Coracoclavicular ligament reconstruction using the lateral half of the conjoined tendon. J Shoulder Elbow Surg. 2004; 13:186-90.

26. Vargas L. Repair of complete acromioclavicular dislocation, utilizing the short head of the biceps. JBJS (Am). 1942; 24:772-3.

27. Weaver JK, Dunn HK. Treatment of acromioclavicular injuries, especially complete acromioclavicular separation. JBJS (Am) 1972; 54(6):1187-94.

28. Gohring U, Matusewicz A, Friedl W, Ruf W. Results of treatment after different surgical procedures for management of acromioclavicular joint dislocation. Chirurg. 1993; 64(7):565-71.

29. Tsou PM. Percutaneous cannulated screw coracolavicular fixation for acute acromioclavicular dislocations. Clin Orthop Relat Res. 1989; 243:112-21.

30. Provencher MT, Clere LL, Romeo AA, Mazzocca AA. Avoiding and managing complications of surgery of the acromioclavicular joint. Book chapter. Springer, 2009, 245-64.

31. Ranne JO, Sarimo JJ, Rawlins MI, Heinonen OJ, Orava S Y. All-arthroscopic double - bundle coracoclavicular ligament reconstruction using autogenous semitendinosus graft: A new technique. Arthrosc Tech. 2012; 1(1):e11-4.

32. Scheibel M, Ifesanya A, Pauly $S$, Haas NP. Arthroscopically assisted coracoclavicular ligament reconstruction for chronic acromioclavicular joint instability. Arch Orthop Trauma Surg. 2008; 128(11):1327-33.

33. Lee SJ, Nicholas SJ, Akizuki KH, McHugh MP, Kremenic IJ, Ben-Avi S. Reconstruction of the coracoclavicular ligaments with tendon grafts: a comparative biomechanical study. Am J Sports Med. 2003; 31:648-55. 